\title{
Treatment Options for Acute Migraine
}

\section{a report by \\ Andrew J Dowson}

Director of the King's Headache Service, King's College Hospital

DOI:10.17925/ENR.2006.00.02.28

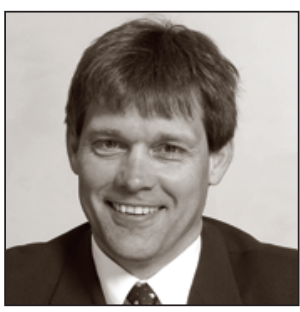

Andrew Dowson is the Director of the NHS King's Headache Service at King's College Hospital, London. He also has several general practice (GP)-based, headache-focused positions. He has served as an investigator in many clinical studies of headache, on epidemiology, medications and devices. He is chairman of Migraine in Primary Care Advisors (MIPCA), the leading UK charity that promotes headache management in primary care, and chairman of the international organisation Headache Care for Practising Clinicians. In recent years he has chaired the Primary Care Neurology Society (P-CNS). Dr Dowson has authored numerous original articles, reviews, books and congress presentations in the field. qualified in medicine in 1984, and worked as a GP for several years before becoming a specialist in headache.

Introduction

Migraine is a common and painful condition that affects approximately $6 \%$ of men and $20 \%$ of women, predominantly from young adulthood to middle age; the years of maximum work and family commitments. ${ }^{1}$ Sufferers experience episodic attacks of moderate to very severe headache (typically throbbing, unilateral and exacerbated by physical activity), accompanied by nausea, photophobia and phonophobia. $^{2}$ Migraine is a highly disabling condition that leads to reduced quality of life and suffering among sufferers. ${ }^{3}$ Attacks are heterogeneous in symptomatology, severity and disability, both between different individuals and between separate attacks in the individual sufferer. ${ }^{4}$

Despite its high prevalence, migraine is generally not well treated in the clinic. Many patients do not consult with a physician, and of those that do, under-diagnosis and under-treatment are both common. ${ }^{3}$ Over the years, a large number of educational initiatives have tried to address these barriers to successful care, although progress has been variable. One of the major breakthroughs was the pioneering work of the US Headache Consortium to provide evidence-based ratings of migraine treatments and guidelines for their use. ${ }^{5}$ This work, and follow-up research on emerging therapies, has allowed the development of evidence-based guidelines for migraine treatments in the USA, ${ }^{5}$ Canada $^{6}$ the UK, ${ }^{7}$ France $^{8}$ and internationally. ${ }^{9}$

This article reviews the migraine treatments that have Grade A evidence of clinical effectiveness and can therefore be recommended for use in the clinic. Grade A clinical evidence requires at least one randomised controlled trial as part of a body of literature of overall good quality and consistency addressing the specific recommendation (evidence levels Ia [evidence obtained from meta-analysis of randomised controlled trials] and Ib [evidence obtained from at least one randomised controlled trial]). ${ }^{5}$
Migraine Treatments

Migraine therapy falls into two broad areas. Acute treatments are used to treat each attack as it occurs and are needed by all patients. Preventive treatments are taken daily by some patients in an attempt to stop the attacks developing. No preventive treatment is $100 \%$ effective, which explains why acute treatments should always be supplied to the patient. Some acute and preventive therapies are available for the patient to buy overthe-counter (OTC) without a prescription, while others (usually the most effective ones) can only be provided by a physician with a prescription.

\section{Acute Migraine Treatments}

The goals of acute treatment are that it should rapidly relieve the headache and other symptoms of migraine and permit the return to normal activities. $^{5,7}$ A time limit of two hours has been suggested for these effects. ${ }^{7}$ Acute medications should be provided for all patients, and patients should be given a portfolio of acute medications to treat attacks of differing severities and provide rescue medication if the initial therapy fails. ${ }^{10}$ Acute treatments with Grade A evidence of effectiveness are shown in Table 1., ${ }^{5,11-15}$

First-line treatments include simple analgesics (paracetamol, ${ }^{12,13}$ aspirin, ${ }^{5}$ non-steroidal antiinflammatory drugs (NSAIDs) ${ }^{5}$ and combination analgesics ${ }^{5}$ ) for mild-to-moderate attacks. Triptans (usually in tablet form) are now the treatments of choice for patients with moderate-to-severe migraine and for those who have failed on analgesic-based therapies. ${ }^{5}$ Sumatriptan, zolmitriptan, rizatriptan, almotriptan and eletriptan have broadly similar clinical profiles and are the usual first-choice triptans. Naratriptan and frovatriptan have a relatively slow onset of action but long plasma half-lives. They may be best suited to patients with long-lasting attacks, headache recurrence (where the headache returns a few hours after initially successful treatment) or those reporting bothersome side effects with the other 


\section{Triptans (POM)}

Subcutaneous injection

\begin{tabular}{llll} 
Sumatriptan & High & Frequent & Severe/refractory migraine and for emergency use \\
\hline Nasal sprays & High & Occasional & \\
Sumatriptan & High & Occasional & Severe/unpredictable attacks and as rescue medication \\
Zolmitriptan"l & & \\
\hline
\end{tabular}

\section{Tablets}

Almotriptan"

(scientific/clinical)

\section{Eletriptan"l}

mildFrovatriptan"

Naratriptan

Rizatriptan

Sumatriptan*

Zolmitriptan

\section{Ergots (POM)}

Dihydroergotamine nasal spray

Simple analgesics (OTC)

$\begin{array}{ll}\text { High } & \text { Infrequent } \\ \text { High } & \text { Occasional } \\ \text { Moderate } & \text { Infrequent } \\ \text { Moderate } & \text { Infrequent } \\ \text { High } & \text { Occasional } \\ \text { High } & \text { Occasional } \\ \text { High } & \text { Occasional }\end{array}$

Moderate-severe attacks. Recommended to be taken early in the attack, when the headache is

Moderate

Occasional Moderate-severe attacks and where headache recurrence is a problem Recommended to be taken as soon as possible after migraine onset,

if possible before the headache starts

\begin{tabular}{llll}
\hline Paracetamol'12,13 & Moderate & Infrequent & Mild-moderate attacks. High dose needed (I,000mg) \\
\hline Aspirin & Moderate & Occasional & Mild-moderate attacks. High dose needed (I,000mg) \\
\hline NSAIDs & & \\
\hline Ibuprofen & Moderate & Occasional & Mild-moderate attacks \\
\hline Naproxen sodium & Moderate & Occasional & \\
\hline
\end{tabular}

Combination analgesics (OTC)

Paracetamol + aspirin + caffeine

Moderate-High

Infrequent

Migraine. Excedrin ${ }^{\circledR}$ (USA), Anadin Extra ${ }^{\circledR}$ (UK)

Analgesics plus anti-emetics (POM)

Aspirin/Paracetamol + metoclopramide ${ }^{14}$ Moderate-High Occasional

Migraine with associated nausea/vomiting

Opiates (POM)

Butorphanol nasal spray

Oral combination opiates

High Frequent

Moderate

Frequent

Severe/refractory migraine and emergency use. Limit use due to potential for habituation

Anti-emetics (POM)

Parenteral metoclopramide ${ }^{15}$

Moderate-High Occasional-Frequent

Emergency use and for refractory nausea/vomiting

POM = prescription only medication; OTC $=$ over-the-counter

* Sumatriptan $50 \mathrm{mg}$ tablets can be sold OTC in the UK to appropriate patients

(adapted and updated from reference 5).

triptans. ${ }^{11}$ Dihydroergotamine nasal spray also has a long duration of action and may be appropriate for patients with long-lasting attacks and/or headache recurrence. ${ }^{5}$ Ergotamine is now not generally recommended for use in migraine due to its suboptimal efficacy profile and the risk of potentially severe side effects. ${ }^{5}$ The combination of analgesics and anti-emetics provides a further option for attacks with associated nausea and/or vomiting, although the triptans are also effective for these symptoms. ${ }^{14}$

Patients who have unpredictable attacks may benefit from the orally disintegrating tablet (ODT) formulations of zolmitriptan and rizatriptan (although these formulations are not absorbed in the mouth), or the nasal spray formulations of sumatriptan and zolmitriptan (again mostly swallowed, but a proportion absorbed via the nasal mucous membranes). Patients with particularly severe attacks, those with a need for rapid response, those with nausea and (especially) vomiting and those attending an emergency room may require nasal spray triptans or subcutaneous sumatriptan. ${ }^{11}$ Parenteral anti-emetics and opiates may also be useful in the emergency situation. ${ }^{5,15}$ However, opiates need to be used with care due to safety concerns and the risk of habituation.

Recent developments illustrate the best way to use triptans in practice and offer more flexible treatment options. Triptan tablets are now recommended for use early in the attack when the headache is mild in intensity. ${ }^{16}$ Many patients have attacks of differing severity and may require more than one acute treatment, while all require rescue 


\section{Drug} Beta-blockers

Propranolol/8

Timolol

Neuromodulators

Topiramate $^{18-20}$

Divalproex sodium/

sodium valproate

Antidepressants

Amitriptyline

Moderate-High
Effectiveness (scientific/clinical) Adverse effects

Occasional

Occasional

Frequent

Frequent

Frequent

Frequent
Recommendations

First-line treatment, especially if hypertension, angina and anxiety are comorbid

First-line treatment, especially if epilepsy

Serotonin antagonists

Methysergide Moderate-High (both drugs), bipolar disorder or $\mathrm{CDH}$
(valproate only) are comorbid

(both drugs), bipolar disorder or CDH
(valproate only) are comorbid

First-line treatment, especially if depression with anxiety or $\mathrm{CDH}$ are comorbid

Specialist use only, as good efficacy is combined with side effect concerns

(adapted and updated from reference 5).

medications. Some will cope well with an analgesic for milder attacks and a triptan for more severe ones. Others may need two different triptans or different formulations of the same triptan, one for initial treatment and one for rescue. Studies show that many patients who fail to respond to one triptan often respond to a different one. ${ }^{11}$ A tablet and a nasal spray (e.g. sumatriptan and zolmitriptan) can be used concurrently. The use of different triptans at the same time is generally contra-indicated, but seems to work in practice. If one triptan fails consistently, the physician should provide another at follow up. In the UK, sumatriptan $50 \mathrm{mg}$ tablets are now approved for sale OTC with a pharmacy prescription, with stringent safeguards put into operation to prevent at-risk patients from receiving the drug. reduction by $\geq 50 \%$ is frequently used as the clinical target. ${ }^{17}$ In clinical studies the best preventive treatments have this effect in about $50 \%$ of patients, confirming that acute medications should also be supplied. A consensus meeting of specialist physicians defined the patients best suited for preventive medications as those who:

- have frequent, high-impact migraine attacks ( $\geq 3-4 /$ month);

- have significant co-morbidities that preclude the use of effective acute medications (also, when one medication can treat both the migraine and the co-morbid condition); and

- overuse acute medications and/or may have CDH. ${ }^{17}$

\section{Migraine is a highly disabling condition that leads to reduced quality of life and suffering among sufferers.}

One caution with all acute medications is the potential for overuse and the development of chronic daily headache $(\mathrm{CDH})$, a condition that is particularly difficult to treat. Patients should be advised not to use acute medications on $\geq 2$ days per week, and those suspected of overuse should be called in for review. ${ }^{7,17}$

\section{Preventive Migraine Treatments}

The clinical goal of preventive medications is to reduce the headache frequency significantly, and a
Preventive treatments with Grade A evidence of effectiveness are shown in Table 2.5,18-20 Clinical evidence favours the use of a beta-blocker (usually propranolol) or the neuromodulators topiramate or valproate as first-line preventive treatments. Betablockers are usually tried first as they have been available for migraine for much longer than the neuromodulators and also seem to have a superior side effect profile. ${ }^{5,18-20}$ However, choice of an appropriate preventive drug can be driven by the drugs' relative co-indications and contraindications (see Table 2). ${ }^{17}$ In some countries, the 
serotonin antagonist pizotifen or a calcium channel blocker (usually verapamil or flunarizine) are used as first-line preventive therapies, even though their clinical profiles are suboptimal. These drugs may be best used as second-line therapies, unless coindications or contra-indications are favourable. For example, pizotifen is the only preventive drug licensed for use in children in the UK, verapamil is co-indicated for hypertension, angina and arrhythmias and flunarizine for vestibular vertigo, insomnia and anxiety. ${ }^{17}$

All the preventive drugs have several contraindications, special precautions for use and associated side effects, which may limit their utility in a selflimiting condition such as migraine. Full prescribing recommendations should be consulted for all these drugs before issuing a prescription.

Several non-prescribed preventive therapies are also available without prescription, and may be attractive to the physician and patient due to their generally non-invasive nature and lack of side effects. ${ }^{7}$

- Behavioural therapies, including relaxation, biofeedback, stress reduction strategies and the avoidance of migraine triggers.

- Physical therapies, including acupuncture, cervical manipulation, massage and exercise.

- Some complementary medications, including feverfew, magnesium, riboflavin, co-enzyme Q and butterbur root. the criteria for Grade A clinical evidence, with the strongest evidence deriving from the butterbur root study. These therapies may be recommended to be used in addition to the patient's existing acute and/or preventive therapies. However, patients should be encouraged to consult accredited complementary practitioners and to only use formulations that have been shown to be effective in the clinical studies cited.

Finally, it is certainly true to say that even the best of the current preventive migraine treatments are suboptimal, and new and more effective treatments are needed.

\section{Conclusion}

Migraine is eminently suited for management in the primary care setting. The gold standard acute and preventive therapies will usually be an oral triptan and a beta-blocker or neuromodulator, respectively. However, treatment needs to be provided that is tailored to each patient's specific needs. For example, someone with a busy lifestyle who perhaps has unpredictable attacks may require treatment that can be 'taken on the move', e.g. an ODT, nasal spray or subcutaneous injection triptan formulation, depending on the severity of the presenting attack. Another person with a predictable lifestyle and attacks will usually do well with a conventional tablet. While analgesic-based therapies can be effective for milder migraine attacks, patients have often exhausted OTC treatments before they ever consult a physician for care. Taking a treatment history will elicit this

\section{The gold standard acute and preventive therapies will} usually be an oral triptan and a beta-blocker or neuromodulator, respectively.

Placebo-controlled studies demonstrate the efficacy of acupuncture, ${ }^{21,22}$ feverfew, ${ }^{23}$ magnesium, ${ }^{24}$ riboflavin, ${ }^{25}$ co-enzyme Q26 and butterbur root. ${ }^{27,28}$ However, of these, only the two acupuncture studies ${ }^{21,22}$ and one study on each of feverfew, ${ }^{23}$ magnesium ${ }^{24}$ and butterbur $\operatorname{root}^{27}$ met information and inform the selection of an effective initial acute treatment. Preventive treatments require persistence on the part of the patient and healthcare professional. Treatment takes a period of months to achieve success and the patient requires support and encouragement during this time.

1. Breslau N, Rasmussen BK, "The impact of migraine: Epidemiology, risk factors, and co-morbidities", Neurology (2001);56(Suppl. 1): pp. 4-12. 
2. Headache Classification Committee of the International Headache Society, "The international classification of headache disorders; 2nd Edition”, Cephalalgia (2004);24(Suppl. 1): pp. 1-160.

3. Lipton RB, Goadsby PJ, Sawyer JPC et al., "Migraine: diagnosis and assessment of disability", Rev Contemp Pharmacother (2000);11: pp. 63-73.

4. Stewart WF, Shechter A, Lipton RB, "Migraine heterogeneity: Disability, pain intensity, and attack frequency and duration”, Neurology (1994);44(Suppl. 4): pp. S24-39.

5. Silberstein SD, for the US Headache Consortium, "Practice parameter: evidence-based guidelines for migraine headache (an evidence-based review). Report of the Quality Standards Subcommittee of the American Academy of Neurology", Neurology (2000); 55: pp. 754-762.

6. Pryse-Phillips WEM, Dodick DW, Edmeads JG et al., "Guidelines for the diagnosis and management of migraine in clinical practice”, Can Med Assoc J (1997);156: pp. 1273-1287.

7. Dowson AJ, Lipscombe S, Sender J et al., "New guidelines for the management of migraine in primary care", Curr Med Res Opin (2002);18: pp. 414-439.

8. Géraud G, Lantéri-Minet $M$, Lucas $C$ et al., "French guidelines for the diagnosis and management of migraine in adults and children", Clin Ther (2004);26: pp. 1305-1318.

9. Dowson AJ, Sender J, Lipscombe $S$ et al., "Establishing principles of migraine management in primary care", Int J Clin Pract (2003);57: pp. 492-507.

10. Lipscombe S, Rees T, Dowson AJ, "Tailoring migraine management in primary care to the needs of the individual patient", Headache Care (2004);1: pp. 147-157.

11. Dowson AJ, Kilminster S, Peters $M$ et al., "Understanding the evidence: evaluating the efficacy of migraine medications in clinical practice", Headache Care (2005);2: pp. 133-143.

12. Lipton RB, Baggish JS, Stewart WF et al., "Efficacy and safety of acetaminophen in the treatment of migraine: results of a randomized, double-blind, placebo-controlled, population-based study", Arch Intern Med (2000);160: pp. 3486-3492.

13. Diener HC, Pfaffenrath V, Pageler L et al., "The fixed combination of acetylsalicylic acid, paracetamol and caffeine is more effective than single substances and dual combination for the treatment of headache: a multicentre, randomized, double-blind, single-dose, placebo-controlled parallel group study", Cephalalgia (2005);25: pp. 776-787.

14. Tfelt-Hansen P, Henry P, Mulder LJ et al., "The effectiveness of combined oral lysine acetylsalicylate and metoclopramide compared with oral sumatriptan for migraine", Lancet (1995);346: pp. 923-926.

15. Colman I, Brown MD, Innes GD et al., "Parenteral metoclopramide for acute migraine: meta-analysis of randomised controlled trials”, BMJ (2004);329: pp. 1369-1373.

16. Dowson AJ, Mathew NT, Pascual J, "Review of clinical trials using early acute intervention with oral triptans for migraine management”, Int J Clin Pract (2006);60: pp. 698-706.

17. Tepper SJ, D'Amico D, Baos V et al., "Guidelines for prescribing prophylactic medications for migraine: a survey among headache specialist physicians in different countries", Headache Care (2004);1: pp. 267-272.

18. Diener HC, Tfelt-Hansen P, Dahlöf $C$ et al., "Topiramate in migraine prophylaxis: results from a placebo-controlled trial with propranolol as an active control”, J Neurol (2004);251: pp. 943-950.

19. Silberstein SD, Neto W, Schmitt J et al., "Topiramate in migraine prevention: results of a large controlled trial", Arch Neurol (2004); 61: pp. 490-495.

20. Brandes J, Saper J, Diamond $M$ et al., "Topiramate for migraine prevention: a randomized controlled trial", JAMA (2004);291: pp. 965-973.

21. Linde K, Streng A, Jurgens $S$ et al., "Acupuncture for patients with migraine: a randomized controlled trial", JAMA (2005);293: pp. 2118-2125.

22. Diener H-C, Kronfeld K, Boewing $G$ et al., "Efficacy of acupuncture for the prophylaxis of migraine: a multicentre randomised controlled clinical trial”, Lancet Neurology (2006); online publication 2 March 2006.

23. Diener HC, Pfaffenrath V, Schnitker J et al., "Efficacy and safety of $6.25 \mathrm{mg}$ t.i.d. feverfew CO2-extract (MIG-99) in migraine prevention - a randomized, double-blind, multicentre, placebo-controlled study", Cephalalgia (2005);25: pp. 1031-1041.

24. Peikert A, Wilimzig C, Kohne-Volland R, "Prophylaxis of migraine with oral magnesium: results from a prospective, multi-center, placebo-controlled and double-blind randomized study", Cephalalgia (1996);16: pp. 257-263.

25. Schoenen J, Jacquy J, Lenaerts $M$, "Effectiveness of high-dose riboflavin in migraine prophylaxis. A randomized controlled trial”, Neurology (1998);50:466-470.

26. Sandor PS, Di Clemente L, Coppola G et al., "Efficacy of coenzyme Q10 in migraine prophylaxis: a randomized controlled trial”, Neurology (2005);64: pp. 713-715.

27. Lipton RB, Gobel H, Einhaupl KM et al., "Petasites hybridus root (butterbur) is an effective preventive treatment for migraine”, Neurology (2004);63: pp. 2240-2244.

28. Diener HC, Rahlfs VW, Danesch U, "The first placebo-controlled trial of a special butterbur root extract for the prevention of migraine: reanalysis of efficacy criteria”, Eur Neurol (2004);51: pp. 89-97. 